[0212-7199 (2005) 22: 12; pp 561-568] ANALES DE MEDICINA Copyright (C) 2005 ARAN EDICIONES, S.L

AN. MED. INTERNA (Madrid) Vol. 22, N. ${ }^{\circ} 12$, pp. 561-568, 2005

\title{
Evolución temporal de las plaquetas y los anticuerpos antiplaquetarios en pacientes de área endémica con malaria no complicada
}

\author{
A. RÍOS-ORREGO, T. ÁLVAREZ-CASTILLO, J. CARMONA-FONSECA'1 , S. BLAIR- \\ TRUJILLO ${ }^{2}$
}

Grupo Malaria. Universidad de Antioquia. Medellín, Colombia

\section{RESUMEN}

Objetivo: evaluar plaquetas y anticuerpos antiplaquetarios IgG Ac-Pl (medidos por citometría de flujo) en pacientes de Turbo (Antioquia, Colombia) con paludismo no complicado.

Metodología: estudio descriptivo, prospectivo y longitudinal de 45 pacientes (14-67 años). Treinta enfermos se siguieron 7 días.

Resultados: A) Pretratamiento: hubo trombocitopenia malárica (TM) en $71 \%$ y Ac-Pl en 33\%, con asociación significativa entre TM y Ac-Pl. La TM no se asocia con zona de residencia, haber tenido malaria en último año ni especie actual de Plasmodium, pero si con sexo (hombres) $(\mathrm{p}=$ 0,02078195), pero la concentración de Ac-Pl es similar en hombres y mujeres. Igualmente, la cantidad de plaquetas es estadísticamente igual entre los valores de las variables anteriores. La presencia de Ac-Pl no se relacionó con las anteriores variables. Hay baja e inversa correlación lineal entre plaquetas y Ac-Pl $(r=-0,342(p=0,02310)$. B) Durante el seguimiento, la regresión lineal múltiple entre plaquetas y edad, años de residencia, número de episodios de malaria en el último año, parasitemia (expresada logarítmicamente) y concentración de Ac-Pl demostró que ningún coeficiente es significativo y la máxima explicación lograda (17\%) la dan la parasitemia y los Ac-Pl. El mismo análisis entre Ac-Pl y edad, años de residencia en la zona, número de episodios de malaria en el último año, parasitemia (expresada logarítmicamente) demostró que las tres primeras explican $38 \%$ de los cambios en Ac-Pl, mientras que los episodios de malaria y el tiempo de residencia en la zona explican el $28 \%$.

PALABRAS CLAVE: Trombocitopenia. Anticuerpos antiplaquetarios. Malaria. Citometría de flujo.
TEMPORAL EVOLUTION OF PLATELETS AND ANTI-PLATELETS ANTIBODIES IN PATIENTS OF ENDEMIC AREA WITH NON COMPLICATED MALARIA

\begin{abstract}
Aim: To evaluate the number of platelets and the title of anti-platelet antibodies IgG Ac-PI (by flow cytometry) in patients with non-complicated malaria.

Methods: Descriptive, prospective and longitudinal study from 45 patients (aged among 14-67). Thirty patients were followed during 7 days.

Results: A) Pretreatment: it was found malarial thrombocytopenia (MT) in $71 \%$ and significant association between MT and Ac-PI in 33 \%. MT was not associated to residence zone, to have had malaria in the last year and Plasmodium species but sex associated (males, $p=$ 0,02078195), although, the Ac-PI titers of male and female was similar. Correspondingly, the number of platelets was statistically similar to the values of above variables. There was a low and an inverse lineal correlation between platelets and titres of Ac-P1 $(r=-0,342(p=0,02310)) . B)$ During the following, multiple linear regression among platelets, age, years of residence in the zone, number of malaria episodes in the last year, parasitaemia (logarithmically expressed) and Ac-P1 titers showed no significant coefficient and the maximum explanation achieved (17\%) was parasitaemia and Ac-P1 titers. Similar analysis among Ac-P1 and age, years of residence in the zone number of malaria episodes in the last year, parasitaemia (logarithmically expressed) showed that the first three explain $38 \%$ of the changes in Ac-P1, whereas malaria episodes and years of residence in the zone explain $28 \%$.
\end{abstract}

KEY WORDS: Thrombocytopenia. Anti-platelets antibodies. Malaria. Flow cytometry.

Ríos-Orrego A, Álvarez-Castillo T, Carmona-Fonseca J, Blair-Trujillo S. Evolución temporal de las plaquetas y los anticuerpos antiplaquetarios en pacientes de área endémica con malaria no complicada. An Med Interna (Madrid) 2005; 22: 561-568.

\section{INTRODUCCIÓN}

La malaria es un grave problema de salud pública en el mundo y en Colombia $(1,2)$ y la trombocitopenia (menos de 150.000 plaquetas $/ \mathrm{mm}^{3}$ (3) ocurre durante esta enfermedad con alta frecuencia (4-9). Plasmodium falciparum y P. vivax se asocian con trombocitopenia malárica (TM), aunque en porcentajes variables y con diferente gravedad.

En pacientes con malaria no tratados, no hay relación de la TM con parasitemia $(10,11)$ ni con la gravedad del paludismo $(6,8,9,11)$. En la India, la TM es un hallazgo común en malaria falciparum pero raro en paludismo vivax (12). Nosotros

Trabajo aceptado: 26 de julio de 2005 
hallamos TM en pacientes no complicados (9) y en pacientes hospitalizados por presentar complicaciones maláricas (8). En pacientes con malaria vivax no complicada hubo $8 \%$ con TM $\mathrm{y}$, entre estos, $25 \%$ mostraron epistaxis y petequias (9), mientras en pacientes con malaria (falciparum o vivax) complicada, hospitalizados, la frecuencia de TM fue $36 \%$, la de hemorragias $4 \%$ y la de ambas fue $3 \%$ (8/291) (8).

Como causa de la TM algunos proponen la destrucción mediada por anticuerpos antiplaquetarios y otros al exagerado proceso de secuestro esplénico, aunque hay informes recientes que indican que el sitio de secuestro plaquetario, en pacientes con malaria no complicada, no es el bazo ni el hígado sino que tal fenómeno ocurre en múltiples sitios (13). Hay destrucción de plaquetas debida a anticuerpos IgG (10-12,1416); este mecanismo es mediado por complejos inmunes circulantes formados por antígenos de Plasmodium e inmunoglobulinas séricas, principalmente Ig G y M (17); se describen tres situaciones: a) presencia de anticuerpos antiplaquetarios (Ac-Pl) con TM $(10,12,15,16)$; b) presencia de Ac-Pl y recuento de plaquetas normal $(10,16)$; c) ausencia de Ac-Pl IgG y presencia de TM (16).

Los macrófagos del bazo - hiperactivos durante la malaria- hacen fagocitosis plaquetaria durante la infección y ella ocurre como consecuencia de la unión de complejos circulantes antígenos de Plasmodium-inmunoglobulinas séricas a las plaquetas (17) y, debido a esto, ellas se activan y experimentan cambios estructurales y bioquímicos que disminuyen sus funciones $\mathrm{y}$, en consecuencia, son reconocidas por el bazo y sacadas de la circulación. La agregación es una función plaquetaria alterada luego de esta activación, por lo que el paciente malárico es más propenso a sangrados $(15,17,18)$.

No es claro el papel de las plaquetas en la gravedad del paludismo, pues hay estudios que las asocian con patogénesis y otros con protección $(19,20,21)$. En cultivos in vitro, las plaquetas inhiben el desarrollo de $P$. falciparum al actuar sobre estadios tempranos del ciclo eritrocítico e impedir la formación de esquizontes (19). En ratones con trombocitopenia inducida por inyección de suero antiplaquetario hay menor frecuencia de malaria cerebral y se considera que la TM impidió la oclusión de la microvasculatura cerebral (20). Los datos sobre el carácter patogénico de las plaquetas en la infección malárica incluyen la demostración de que ellas se adhieren a las células endoteliales de la microvasculatura cerebral donde son secuestradas y favorecen la obstrucción de los vasos y el desarrollo de la malaria cerebral $(17,21)$, fenómeno que puede estar mediado por el factor de necrosis tumoral alfa (FNT- $\alpha$ ) $(20,22,23)$. Por otra parte, en pruebas in vitro, se han puesto en contacto eritrocitos infectados con $P$. falciparum y, por otro lado, plaquetas de individuos sanos; se encontró que la presencia de la glicoproteína de membrana CD36 en la superficie plaquetaria está implicada en la aglutinación de eritrocitos parasitados, considerándose este hecho como un mecanismo de patogénesis de la enfermedad $(24,25)$.

La TM suele ser independiente de la gravedad de la enfermedad y rara vez se asocia con hemorragias o coagulación intravascular diseminada $(10,26)$. Hay asociación entre TM y factores como fiebre mayor de $39{ }^{\circ} \mathrm{C}$, transaminasas aumentadas y altos niveles de parasitemia, pero no con sexo, edad y quimioprofilaxis (7); además, se relaciona con leucopenia y anemia (27) y variables inmunitarias (concentración de complejos inmunes circulantes, activación de complemento) $(10,28)$. En pacientes palúdicos ambulatorios hemos hallado: a) TM en $35 \%$ de los menores de 16 años y $49 \%$ en mayores de 15 años; b) mayor frecuencia de TM por $P$. vivax en menores de 16 años, mientras que en mayores de 15 años ambas especies tuvieron frecuencia similar; c) la parasitemia y la TM no se asociaron, d) la TM se distribuyó igualmente por sexos (5).

El presente estudio evalúo el comportamiento temporal de las plaquetas y los Ac-Pl tipo IgG durante la enfermedad malárica no complicada, así como la relación entre plaquetas y Ac-Pl con variables clínico-epidemiológicas. También se evaluó la asociación de TM con especie parasitaria ( $P$. vivax, $P$. falciparum) y con Ac-Pl tipo IgG.

\section{MATERIAL Y MÉTODOS}

\section{TIPO DE ESTUDIO, POBLACIÓN DE REFERENCIA Y DISEÑO DE LA MUESTRA}

Se aplicó un diseño descriptivo, longitudinal y prospectivo. La población de referencia son los pacientes ambulatorios, mayores de 14 años, de cualquier sexo, con paludismo por $P$. vivax o $P$. falciparum, sin complicación según los criterios de la Organización Mundial de la Salud OMS (29) —acogidos por el Ministerio de Protección Social de Colombia (30) residentes en zona rural o urbana del municipio de Turbo (Antioquia Colombia), y que consultaron al puesto de diagnóstico de malaria de este municipio.

La muestra se diseñó y calculó teniendo en cuenta una población de 2000 pacientes con malaria mayores de 14 años (2); se aplicaron una frecuencia esperada de TM de $8 \%$ (9), un nivel de confianza de $95 \%$, un error de muestreo de $10 \%$, lo que llevó a un tamaño muestral de 28 pacientes, captados según el orden de llegada entre el 21 de mayo y el 27 de junio de 2001, si cumplían con los criterios de inclusión. Captamos 45 y 30 se siguieron por 7 días para evaluar el comportamiento temporal de los trombocitos. Cada paciente que aceptó participar en el estudio firmó un consentimiento informado, aprobado por el comité de ética del Centro de Investigaciones Médicas de la Universidad de Antioquia.

\section{CRITERIOS DE INCLUSIÓN Y EXCLUSIÓN}

Inclusión: gota gruesa positiva para $P$. falciparum o $P$. vivax, cualquier valor de parasitemia menor de $50.000 / \mu \mathrm{L}$, más de 14 años, no tener embarazo ni complicaciones de malaria, vivir en Turbo, comprometerse a asistir a mínimo tres controles, aceptar participar en la investigación y firmar el consentimiento informado.

Exclusión: requerir hospitalización o presentar malaria clínicamente complicada según los criterios clínicos de la OMS (29), faltar a dos controles o al control del día 3.

\section{DIAGNÓSTICO Y TRATAMIENTO DE LA MALARIA}

El diagnóstico se hizo con gota gruesa y la parasitemia en sangre periférica se midió según las pautas de la OMS (31). El tratamiento para los pacientes palúdicos se hizo según criterios del Ministerio de Protección Social de Colombia (30). 


\section{SEGUIMIENTO DE LOS PACIENTES}

Antes del tratamiento, a cada uno de los 45 pacientes se le hicieron recuentos de parásitos y de plaquetas y medición de la concentración de Ac-Pl IgG. Treinta de los 45 pacientes captados fueron seguidos durante 7 días y las mismas mediciones se realizaron los días 2 (48 horas de iniciado el tratamiento antimálarico), 3 (72 horas de iniciado el tratamiento y 24 horas de haber terminado el esquizonticida circulante) y el día 7 (cuatro días después de terminado el tratamiento con el esquizonticida circulante).

\section{RECUENTO DE PLAQUETAS}

Se recolectaron $5 \mathrm{~mL}$ de sangre venosa periférica en tubos al vacío con EDTA para el recuento plaquetario, hecho con contador electrónico de partículas (Bayer Advia 60 TC), aproximadamente 4 horas después de tomada la muestra. Los valores de referencia, según el laboratorio, fueron 150.000 a $400.000 / \mathrm{mm}^{3}$.

\section{DETERMINACIÓN DE ANTICUERPOS ANTIPLAQUETARIOS IGG POR CITOMETRÍA DE FLUJO}

\section{Toma de muestras}

Se obtuvieron $7 \mathrm{~mL}$ de sangre venosa, en tubos al vacío sin anticoagulante; se centrifugaron a $3.600 \mathrm{rpm}$ por $10 \mathrm{minu}-$ tos y el suero fue almacenado en viales plásticos de $1,8 \mathrm{~mL}$ y congelado a $-20{ }^{\circ} \mathrm{C}$ hasta llegar a Medellín, donde se congelaron a $-70{ }^{\circ} \mathrm{C}$ hasta su lectura en el citómetro de flujo, aproximadamente 4 meses después.

\section{Sueros controles}

Suero control positivo: se usó una mezcla de sueros de 20 personas adultas de grupo sanguíneo AB sensibilizados contra antígenos HLA. Los sueros fueron suministrados por el grupo de investigación "Inmunología celular e inmunogenética" de la Universidad de Antioquia.

Suero control negativo: mezcla de suero de 6 hombres sanos de grupo sanguíneo $\mathrm{AB}$, no transfundidos. El suero fue inactivado con calor a $56{ }^{\circ} \mathrm{C}$, por una hora, para medir anticuerpos citotóxicos, IgG e IgM por citometría de flujo, los cuales estuvieron ausentes.

\section{Preparación de plaquetas para determinar los anticuerpos antiplaquetarios en suero}

Se tomaron $5 \mathrm{~mL}$ de sangre venosa en tubos al vacío con EDTA de cuatro hombres adultos sanos, de grupo sanguíneo "O". Las plaquetas fueron purificadas de los eritrocitos y leucocitos mediante centrifugaciones y lavadas con tampón Tyrodes (preparado según Ault \& Mitchell) (32), luego fueron fijadas según los métodos de Ault \& Mitchell (32) y de Robinson y colaboradores (33) y, finalmente, se lavaron de nuevo y se refrigeraron a $4{ }^{\circ} \mathrm{C}(32,33)$.

\section{Medición de anticuerpos antiplaquetarios $\operatorname{Ig} G$}

Ochocientas mil plaquetas por $\mathrm{mL}$, lavadas y fijadas, fueron colocadas en viales plásticos de $1,8 \mathrm{~mL}$ y a cada uno se le adicionaron $30 \mu \mathrm{L}$ de suero control positivo, $30 \mu \mathrm{L}$ de suero control negativo y $30 \mu \mathrm{L}$ del suero de cada paciente y se incubaron por 30 minutos a $37^{\circ} \mathrm{C}$. Luego, cada muestra fue lavada dos veces con Tyrodes tampón centrifugando a $2.800 \mathrm{rpm}$ por 5 minutos a $4 \infty \mathrm{C}$, se descartó el sobrenadante y se adicionó $1 \mathrm{~mL}$ del mismo tampón entre cada lavado. Posteriormente cada muestra fue incubada con $5 \mu \mathrm{L}$ de anti-CD41a antihumano marcado con ficoeritrina (Becton Dickinson Company Pharmingen cat 555467 was. $31085 \mathrm{x}$ ) y $10 \mu \mathrm{L}$ de una dilución $1: 10$ de inmunoglobulina antihumana de cabra fragmento especifico (Fab')2 marcado con FITC (isotiocianato de fluoresceína) (Jackson Immunoresearch Laboratories Inc., code 109-096-098 lot: 48911) por 30 minutos a $4{ }^{\circ} \mathrm{C}$ en la oscuridad. Después las muestras fueron lavadas 2 veces como se indico antes y finalmente fueron resuspendidas en un tampón para citometría (PBS/azida 0,1\%/BSA al 1\%) hasta su lectura (32).

Las muestras fueron analizadas en un equipo Coulter EPICS XL (Coulter-Beckman, Miami, Fl, USA) con parámetros: FSC, SSC, FL1 (FITC), FL2 (PE), FL3 (Cy-Chrome). Se hizo una región para separar la población de plaquetas, por tamaño (FSC) y granularidad (SSC), en escalas logarítmicas. Los resultados fueron analizados con el programa winMDI 2.8 (Scripps Research Institute, La Jolla, Ca, USA).

Las medianas de los controles negativos se sumaron y se obtuvo el promedio aritmético de ellas, que resultó en 139 canales de fluorescencia (CF). También se calculo la desviación estándar de las medianas con respecto a la media obtenida, que fue de 17.5 CF. Los títulos por encima del límite definido por la media más 3 desviaciones estándar $(139+52.4)$ se consideraron positivos; es decir, un título de 191 o más CF fue positivo.

\section{ANÁLISIS ESTADÍSTICO}

El análisis de los resultados se hizo con los programas EpiInfo 6.04 y Statgraphcis 7.1. Con el segundo se hicieron estadística descriptiva, pruebas t para muestras independientes, análisis de varianza y de correlación y regresión. Las pruebas de chi cuadrado, incluyendo la versión de Fisher, se realizaron en EpiInfo 6.0. Los gráficos se hicieron con el programa Excel.

En el análisis de varianza (anova): a) las sumas de cuadrados se obtuvieron por el procedimiento de tipo III para tales sumas y todas las razones $\mathrm{F}$ están basadas en el error residual de mínimos cuadrados; b) las diferencias entre las medias y los intervalos de confianza se obtuvieron siempre con la prueba HSD de Tukey; c) en el análisis de rango múltiple siempre se usó el método de Newman-Keuls.

Todas las decisiones sobre significación estadística se tomaron con un nivel de significación menor del $5 \%(\mathrm{p}<0,05)$.

\section{RESULTADOS}

\section{CARACTERÍSTICAS GENERALES DE LOS PACIENTES}

De los 45 pacientes, $58 \%$ fueron hombres, la edad promedio fue 33 años, $64 \%$ vivía en zona rural, el tiempo promedio de residencia en el lugar fue 7,5 años, 36\% sufrió malaria en 
TABLA I

DISTRIBUCIÓN DE 45 PACIENTES CON MALARIA SEGÚN TROMBOCITOPENIA (TM), ANTICUERPOS ANTIPLAQUETARIOS (ACPL) Y ESPECIE DE PLASMODIUM

Parte A. Casos con trombocitopenia malárica (TM) según la presencia de anticuerpos antiplaquetarios y la especie de Plasmodium (a)

Especie

Con AcPl

$\operatorname{Sin} \mathrm{ACPl}$

\begin{tabular}{l|ccc|ccrc}
\hline & Si TM & No TM & Total & Si TM & No TM & Total & Total especie \\
P. falciparum & $6(13)$ & $1(2)$ & $7(15)$ & $6(13)$ & $6(13)$ & $12(27)$ & $19(42)$ \\
P. vivax & $8(18)$ & $0(0)$ & $8(18)$ & $12(27)$ & $6(13)$ & $18(40)$ & $26(58)$ \\
Total & $14(31)$ & $1(2)$ & $15(33)$ & $18(40)$ & $12(27)$ & $30(37)$ & $45(100)$ \\
Grupo & AcPl(+) & AcPl(+) & & AcPl(-) & AcPl(-) & \\
& TM(+) & TM(-) & & TM(+) & TM(-) & \\
\hline
\end{tabular}

(a) entre paréntesis, el porcentaje con respecto a 45 pacientes.

Parte B. Distribución porcentual de la trombocitopenia malárica (TM), los anticuerpos antiplaquetarios (AcPI) y de ambos eventos en los 45 pacientes (b)

\begin{tabular}{cccccc} 
Día & TM(+) & AcPI (+) & AcPI (+) y TM(+) & TM(+) /ACPI(+) & $T M(+) / A C P I(-)$ \\
\hline 1 & $71 \%(32)$ & $33 \%(15)$ & $31 \%(14)$ & $93 \%(43)$ & $60 \%(27)$ \\
3 & $74 \%(33)$ & $49 \%(22)$ & $29 \%(13)$ & $60 \%(27)$ & $89 \%(40)$ \\
4 & $40 \%(18)$ & $42 \%(19)$ & $18 \%(8)$ & $38 \%(17)$ & $40 \%(18)$ \\
\hline
\end{tabular}

(b) entre paréntesis, el número de casos entre el total de 45 pacientes.

el último año y el promedio de episodios maláricos en el último año fue de 2.

La prevalencia de infección fue $58 \%$ (26/45) por $P$. vivax y $42 \%$ (19/45) por P. falciparum. El promedio de parasitemia fue 11.511 parásitos/ $\mu \mathrm{L}$ para $P$. falciparum y 6.522 para $P$. vivax. La frecuencia de TM fue 77\% (20/26) entre los infectados por $P$. vivax y $63 \%(12 / 19)$ en $P$. falciparum. El predominio de $P$. vivax es claro (Tabla I).

Los Ac-Pl fueron positivos en $31 \%(8 / 26)$ de los pacientes con malaria vivax y en $37 \%(7 / 19)$ de aquellos con malaria falciparum. El $100 \%$ de los infectados con $P$. vivax (8/8) y que tenían Ac-Pl positivos, presentaron TM y el $86 \%$ de quienes tuvieron $P$. falciparum y presentaron Ac-Pl desarrollaron TM. Además, 66\% de los enfermos (12/18) con $P$. vivax presentaron TM y no tuvieron Ac-Pl y esta misma característica estuvo asociada a $P$. falciparum en $50 \%(6 / 12)$ (Tabla I).

En general, las plaquetas oscilaron entre $27.000 \mathrm{y}$ $287.000 / \mathrm{mm}^{3}$ (promedio 127.946, mediana 126.000, desviación estándar 58.851). El promedio de plaquetas fue 145.526 y $115.115 / \mathrm{mm}^{3}$ para $P$. falciparum y $P$. vivax, respectivamente. La concentración promedio de Ac-Pl fue $188 \mathrm{CF}$ en los pacientes con $P$. falciparum y de $167 \mathrm{CF}$ en aquellos con $P$. vivax. En ninguna de las comparaciones anteriores hubo diferencia significativa y esto persiste en todos los días de tratamiento. Ninguno de los pacientes presentó manifestaciones hemorrágicas. El comportamiento temporal de plaquetas y de Ac-Pl es similar en ambas especies de parásito y que las tendencias de plaquetas y anticuerpos son contrarias (Fig. 1).

El $85 \%$ de los hombres y el $53 \%$ de las mujeres presentó TM y, entre los pacientes con ella, 69\% fueron hombres (Chi cuadrado Mantel-Haenszel $=5,34 ; p=0,02078195)$. La frecuencia de TM es estadísticamente similar en todo grupo de edad (14-20, 21-30, 31-40, 41-50, 51-67 años), aunque en el grupo menor es $89 \%(8 / 9)$ y en los demás oscila entre 64 y $67 \%$.

\section{RELACIÓN ENTRE TROMBOCITOPENIA Y ANTICUERPOS ANTIPLAQUETARIOS SEGÚN TIEMPO DE EVOLUCIÓN}

Antes de iniciar el tratamiento antimálarico, $71 \%$ (32/45) presentó TM, solo 33\% (15/45) tuvo Ac-Pl y ambos fenómenos coexistieron en $32 \%$ (14/45). El 29\% (13/45) de los pacientes no presentó TM y, entre ellos, $92 \%$ carece de Ac-P1. Existe asociación entre la presencia de TM y de Ac-Pl positi$\operatorname{vos}(\geq 191)$ : la proporción de TM es mayor entre quienes poseen Ac-Pl (93\%: 14/15) que entre quienes no los tienen (60\%: 18/30). Este día 1, la concentración promedio de plaquetas fue de 127. 956/ $\mathrm{mm}^{3}$ (Fig. 1).

A las 48 horas de iniciado el tratamiento antimalárico la situación es distinta: 48\% (15/31) de los pacientes tienen AcPl positivos, $74 \%$ (23/31) tienen TM y 29\% (9/31) posee ambos eventos. La proporción de TM entre los carentes de Ac-Pl (88\%: 14/16) es mucho mayor que entre quienes los tienen (60\%: 9/15), aunque la diferencia no es significativa. Este día, todavía hay déficit de plaquetas, pues el promedio solo es de 125.636 células $/ \mathrm{mm}^{3}$ (Fig. 1).

A las 72 horas de tratamiento (24 horas luego de haber terminado de tomar la cloroquina), el promedio de plaquetas es $162.000 / \mathrm{mm}^{3}, 43 \%$ (13/30) tiene Ac-Pl, 40\% (12/30) presenta TM y $17 \%$ (5/30) tiene ambos eventos; la proporción de $\mathrm{TM}$ en personas con anticuerpos positivos $(38 \%: 5 / 13)$ es similar a las que carecen de ellos (41\%: 7/17) (Chi cuadrado Mantel- Haenzel tiene $\mathrm{p}=0,882434$ ) (Fig. 1).

Cuatro días después de haber terminado el tratamiento el $100 \%$ de las personas tiene recuento normal de plaquetas (promedio: 289.080 plaquetas $/ \mathrm{mm}^{3}$ ), 61\% (14/23) carece de Ac-Pl y 39\% (9/23) posee plaquetas normales y tiene Ac-Pl positivos (Fig. 1).

El comportamiento de las plaquetas en función del tiempo es estadísticamente igual entre quienes poseen Ac-Pl y quienes carecen de ellos (Fig. 2). 


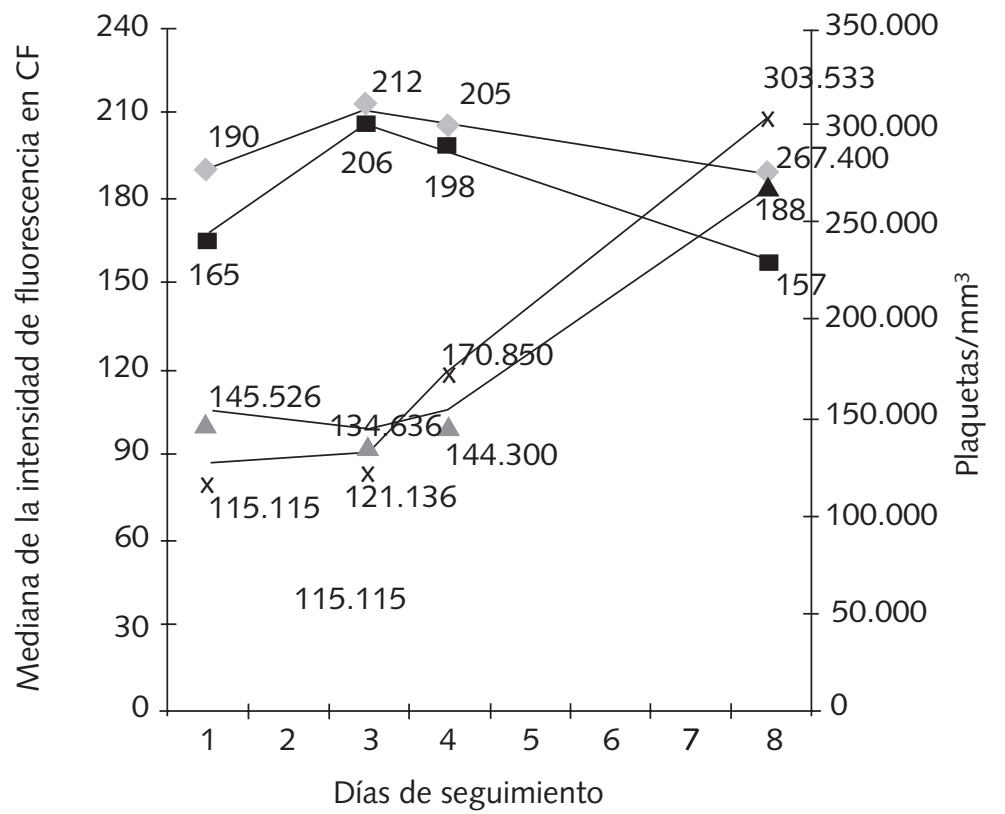

Ac-antiplaq. P. falciparum

Ac-antiplaq. P. vivax

Plaquetas $/ \mathrm{mm}^{3}$ p. falciparum

$\times$ Plaquetas $/ \mathrm{mm}^{3}$ P. vivax

P. falciparum (a)

P. $\operatorname{vivax}(a)$

\begin{tabular}{|c|c|c|c|c|c|c|}
\hline Día & Plaquetas/ $\mathrm{mm}^{3}$ & $\mathrm{Ac}-\mathrm{PI}(\mathrm{CF})$ & Parásitos/ mm $\mathrm{mm}^{3}$ & Plaquetas/ $\mathrm{m}^{3}$ & $A c-P I(C F)$ & Parásitos/ $\mathrm{mm}^{3}$ \\
\hline 1 & $\begin{array}{r}145.526 \\
\pm 67.593\end{array}$ & $165 \pm 79$ & $\begin{array}{c}11.511 \pm \\
18.730\end{array}$ & $\begin{array}{r}115.115 \\
\pm 67.593\end{array}$ & $190 \pm 92$ & $6.521 \pm 704$ \\
\hline 3 & $\begin{array}{r}134.636 \\
\pm 94.429\end{array}$ & $206 \pm 113$ & $589 \pm 1.770$ & $\begin{array}{r}121.136 \\
\pm 55.258\end{array}$ & $212 \pm 142$ & $32 \pm 109$ \\
\hline 4 & $\begin{array}{r}144.300 \\
\pm 86.041\end{array}$ & $198 \pm 107$ & $8 \pm 25$ & $\begin{array}{l}170 \pm 850 \\
\pm 63.305\end{array}$ & $205 \pm 140$ & 0 \\
\hline 7 & $\begin{array}{l}267.400 \\
\pm 78.080 \\
\end{array}$ & $157 \pm 71$ & 0 & $\begin{array}{c}303.533 \\
\pm 109.873\end{array}$ & $188 \pm 129$ & 0 \\
\hline
\end{tabular}

(a) promedio \pm desviación estándar

Fig. 1. Distribución temporal de las plaquetas (Plaq), los anticuerpos antiplaquetarios lgG (Ac-Pl) y la parasitemia, según especie de Plasmodium, en 30 pacientes con malaria no complicada.

En resumen (Tabla I), el porcentaje de pacientes con trombocitopenia es de $71 \%$ antes de iniciar el tratamiento, el día 3 presenta un valor de $74 \%$, el día 4 cae a $40 \%$ y el día 7 es cero. Los Ac-Pl positivos están presentes en $33 \%$ de los casos antes de comenzar la terapia antimalárica y los otros días los presentan entre 42 y $48 \%$ de los pacientes y la concentración (cantidad) de los Ac-Pl IgG es temporalmente estable. Considerando el tiempo, el recuento plaquetario varía en forma significativa únicamente en los extremos (antes del tratamiento y el día 7), pero no hay diferencia entre los otros días. Por otra parte, la concentración diaria de anticuerpos antiplaquetarios es estadísticamente igual en función de los cuatro días de control. No existió diferencia entre las variables plaquetas y Ac$\mathrm{Pl}$ por especie parasitaria en ninguno de los cuatro días de evolución. Según lo anterior, en este estudio el tiempo de seguimiento, que fue corto, es una variable sin importancia en el comportamiento de la trombocitopenia con relación a los anticuerpos antiplaquetarios y, en adelante, se omitirá en el análisis de los resultados.

\section{RELACIÓN DE PLAQUETAS Y ANTICUERPOS ANTIPLAQUETARIOS CON LAS VARIABLES EPIDEMIOLÓGICAS INCLUIDAS EN EL ESTUDIO}

Antes del tratamiento antimalárico, la TM (sí, no) no se asocia con zona de residencia, tener malaria en el último año ni especie plasmodial, pero sí con sexo (Chi cuadrado MantelHaenszel $=5,34 ; p=0,02078195)$. Igualmente, las medianas de plaquetas son estadísticamente iguales entre las categorías de cada una de las variables anteriores. La TM es más frecuente en los hombres, pero la concentración de Ac-Pl es esta- 


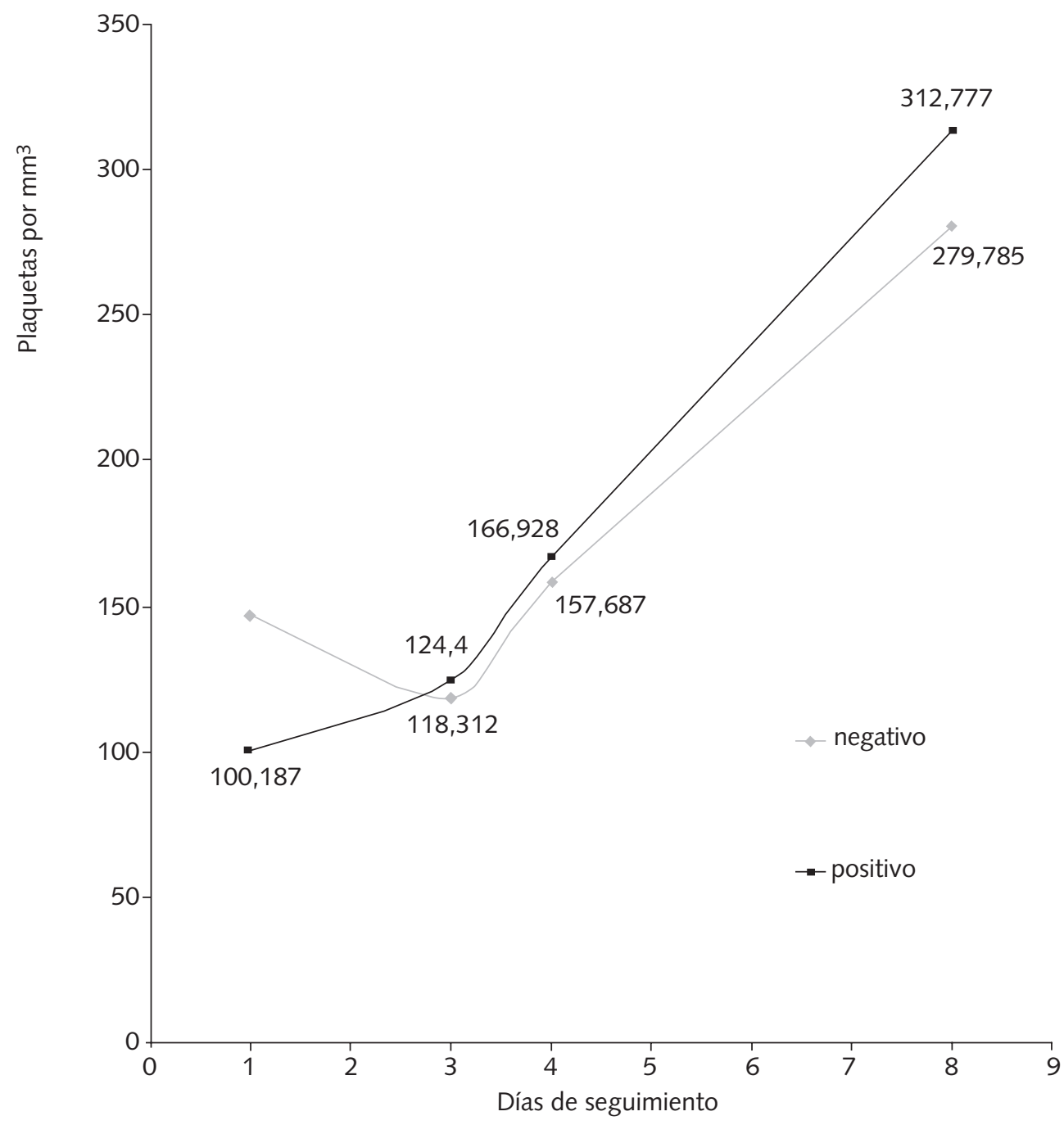

Fig. 2. Evolución temporal de las plaquetas según la presencia de anticuerpos antiplaquetarios, en 30 pacientes con malaria.

dísticamente similar según el sexo (hombres: $174 \mathrm{CF}$ y mujeres: $183 \mathrm{CF}$ ).

La parasitemia tiene inversa y baja pero significativa correlación (coeficiente " $r$ " vale entre 0,307 y 0,342 ) con Ac$\mathrm{Pl}$, pero no existe con edad, tiempo de residencia en el lugar, episodios de malaria en el último año, parasitemia y concentración de Ac-Pl.

\section{COMPARACIÓN DE LOS GRUPOS DE PACIENTES CON TROMBOCITOPENIA Y ANTICUERPOS}

Los grupos indicados en la Tabla I (TM+, AcPl+ ; TM+, AcPl-; TM-, AcPl+; TM-, AcPl-) se compararon según las variables epidemiológicas ya referidas y, excepto el sexo (chi cuadrada $=8,56530) ; \mathrm{p}=0,0356652)$, ninguna presentó asociación.

Un análisis de regresión lineal múltiple (RLM) entre el recuento plaquetario y las variables edad, años de residencia en el lugar, número de episodios de malaria en el último año, parasitemia (expresada logarítmicamente) y concentración de Ac-Pl) demostró que ninguno de los coeficientes de regresión era significativo y la máxima explicación que se logró del comportamiento plaquetario era del $17 \%$, a cargo de parasitemia y concentración de Ac-Pl.

El análisis RLM entre concentración de Ac-Pl y las variables edad, años de residencia en la zona, número de episodios de malaria en el último año, parasitemia expresada logarítmicamente demostró que las tres primeras dabann cuenta del $38 \%$ de los cambios en la concentración de anticuerpos, mientras que los episodios de malaria y el tiempo de residencia en la zona explicaban el $28 \%$.

\section{DISCUSIÓN}

En este estudio con pacientes palúdicos no complicados se encontró TM en $71 \%$ de ellos antes de iniciar el tratamiento antimalárico, valor similar al encontrado en literatura extranjera $(6,7,10,34,35,36)$ y muy superior al informado antes por 
nosotros (9), que fue de $8 \%$ en malaria vivax (ahora fue $77 \%$ en $P$. vivax y $63 \%$ en $P$. falciparum). El recuento de plaquetas volvió a ser normal entre días 3 y 7 días después de iniciado el tratamiento, cualquiera fuese la especie, semejante a los 8 días informados por Bouree \& Torossian (7).

En humanos y en modelos animales, la TM es una manifestación muy temprana que aparece incluso antes que los parásitos $(37,38)$ y ha sido propuesta como factor predictor de la enfermedad (27,39-41); por ello, ante la sospecha de malaria y con la presencia de trombocitopenia y de una gota gruesa negativa, se deberá insistir en buscar los parásitos (repetir la gota gruesa, usar técnicas moleculares), sin olvidar que un recuento plaquetario bajo puede deberse en parte a falsa trombocitopenia (42).

Aunque en nuestro estudio la parasitemia no tuvo relación significativa con la presentación de TM, el comportamiento de las plaquetas fue inversamente proporcional a la parasitemia, hallazgo similar al de Bouree \& Torossian (7).

La TM se relacionó con ambas especies de Plasmodium, como en otros informes $(34,35)$, pero existen datos sobre su fuerte predominio en $P$. falciparum $(12,17,43)$. Aunque según la literatura, la TM no se asocia con sexo ni con edad (44), nosotros la encontramos más frecuente entre hombres y en los de menor edad (14-20 años).

Las variables con mayor poder explicativo (38\%) del comportamiento de los Ac-Pl fueron la edad, los años de residencia en la zona y el número de episodios de malaria; estas variables presentan correlación entre ellas.

La concentración plaquetaria en los pacientes con TM por $P$. vivax mostró curso temporal ascendente, mientras que en aquellos con $P$. falciparum hubo disminución del recuento el día 2 y, además, tardaron más en recobrar el nivel normal de plaquetas. Este hallazgo puede estar asociado con la propiedad procoagulante que ha caracterizado a la enfermedad malárica por $P$. falciparum o a la participación que se le ha atribuido a las plaquetas en la formación de acúmulos de eritrocitos parasitados $(24,25,45)$.

Un hallazgo importante consistió en la fuerte relación entre tener Ac-Pl y presentar TM. En los pacientes que no han recibido tratamiento antimalárico y poseen Ac-Pl, independientemente de su concentración, la TM podría explicarse por la presencia de estos anticuerpos, pero no necesariamente el paciente con TM tiene Ac-Pl, por lo que la presencia de Ac-Pl es una causa de destrucción plaquetaria pero evidente que no es la única y se han postulado otros $(14,44,46)$. También se piensa que los trombocitos están disminuidos en la malaria porque median la aglutinación de eritrocitos infectados en la microvasculatura periférica y cerebral (47). Cabe resaltar que la relación entre la TM y los Ac-Pl en nuestro estudio no se mantiene en el tiempo, porque la concentración de los anticuerpos permaneció constante, mientras la TM comenzó a resolverse en cuanto se inició el tratamiento antimalárico.

La concentración plaquetaria estuvo significativa e inversamente relacionada con la parasitemia y la concentración de Ac-Pl IgG (con más parásitos y anticuerpos, menos plaquetas). Esta relación ocurre solo antes del tratamiento antimalárico y el día 7 , cuando se ha terminado el tratamiento, esta relación cambia (hay pacientes con Ac-Pl pero no existen parásitos ni TM), lo que sugeriría que el medicamento podría estar involucrado con la presencia de anticuerpos antidrogas y que tales anticuerpos podrían unirse a las plaquetas y promover su destrucción, como ya se ha descrito en medicamentos como quinina, amodiaquina y sulfadoxina-pirimetamina $(3,48,49)$.

También podrían atribuirse la presencia de Ac-Pl y un recuento normal de plaquetas a la acción del fármaco y la subsiguiente desaparición de los parásitos sanguíneos, lo que llevaría a una producción eficaz de trombocitos, ocurriendo un estado trombolítico compensado. Esto podría explicar el hecho de que el día 7 ningún paciente presente TM aunque los Ac-Pl están en un porcentaje incluso mayor que antes del tratamiento. En 9 pacientes con malaria cerebral hubo 7 que, inmediatamente después de la terapia con quinina, incrementaron los niveles de inmunoglobulina y de complejos inmunes circulantes y en cuatro esto coincidió con el desarrollo de complicaciones y coma (28).

En nuestro estudio, los pacientes fueron incluidos con el criterio de que fueran ambulatorios, suponiendo que eso significa no tener malaria complicada, pero no estamos seguros de que la TM no sea una complicación ni un factor determinante para desarrollar complicaciones, ya que se ha identificado como signo muy común en pacientes hospitalizados con diagnóstico de malaria grave $(8,12,50,51)$.

En los pacientes maláricos y con trombocitopenia grave (menos de 150.000 plaquetas/mm3), la transfusión de trombocitos es práctica muy común, pero hay que preguntarse si es benéfica, debido a que la TM puede ser de origen inmunitario (10-12,14-16) y, además, porque al transfundir plaquetas se produce sensibilización que aumenta la producción de Ac-Pl que destruirían más plaquetas.

\section{AGRADECIMIENTOS}

A la Universidad de Antioquia por la financiación del proyecto y al grupo de investigación "Inmunología celular e inmunogenética" de la Universidad de Antioquia por el apoyo académico y técnico.

\section{Bibliografía}

1. Carmona-Fonseca J. La malaria en Colombia, Antioquia y las zonas de Urabá y Bajo Cauca: panorama para interpretar la falla terapéutica antimalárica. Parte 1. Iatreia 2003; 16: 299-318.

2. Carmona-Fonseca J. La malaria en Colombia, Antioquia y las zonas de Urabá y Bajo Cauca: panorama para interpretar la falla terapéutica antimalárica. Parte 2. Iatreia 2004;17:34-53.

3. Greer JP, Foerster J, Lukens JN, Rogers GM, Paraskevas F, Glader BE. Wintrobe's Clinical Hematology, 11 e. 2 vol. Philadelphia: Lippincott Williams Wilkins; 2003. Vol 1, p. 1079.

4. Bashwari LA, Mandil AM, Bahnassy AA, Al-Shamsi MA, Bukhari AH.

Epidemiological profile of malaria in a hospital in the eastern region of Arabia. Saudi Med J 2001; 22: 133-8.

5. Sitalakshmi S, Srikrishna A, Devi S, Damodar P, Mathew T, Varghese J. Changing trends in malaria a decade's experience at a referral hospital. Indian J Pathol Microbiol 2003; 46: 399-401.

6. Richards MW, Behrens RH, Doherty JF. Short report: Hematologic changes in acute, imported Plasmodium falciparum malaria. Am J Trop Med Hyg 1998; 59: 859.

7. Bouree P, Torossian A. Thrombocytopenia during malaria. Study of 242 cases. Presse Med 1994; 23: 449. 
8. González L, Guzman M, Carmona J, Lopera T, Blair S. Características clínico-epidemiológicas de 291 pacientes hospitalizados por malaria en Medellín. Acta Med Col 2000; 25: 63-70.

9. Echeverri M, Tobón A, Álvarez G, Carmona J, Blair S. Clinical and laboratory findings of Plasmodium vivax malaria in Colombia, 2001. Rev Inst Med Trop Sao Paulo 2003; 45 (1): 29-34.

10. Touze JE, Mercier P, Rogier C. Platelet activity in malaria thrombocytopenia. Phatology Biology 1990; 38: 678-81.

11. Ohtaka M, Ohyashiki K, Iwa Buchi H, Iwaabuchi A, Lin KY, Toyama $\mathrm{K}$. A case of vivax malaria with thrombocytopenia suggesting immunology mechanism. Rinsho Ketsueki (resumen) 1993; 34: 490-92.

12. Kakar A, Bhoi S, Prakash V, Kakar S. Profund Thrombocytopenia in Plasmodium vivax malaria. Diagn Microbiol Infect Dis 1999; 35: 243-4.

13. Karanikas G, Zedwitz-Liebenstein K, Eidherr H, Schuetz M, Sauerman $\mathrm{R}$, Dudczak $\mathrm{R}$ et al. Platelet kinetics and scintigraphic imaging in thrombocytopenic malaria patients. Thromb Haemost. 2004; 91: 553-7.

14. Kelton J.G, Inmune-mediated thrombocyytopenia of malaria. J Clin Inv 1983; 71: 832-6.

15. Mohanty D, Marwaha N, Ghosh K, Sharma S, Garewal G, Shah S et al. Functional and ultrastructural changes of platelets in malaria infection. Trans R Trop Med Hyg 1988; 82: 369-75

16. Yamaguchi S, Kubota T, Yamagishi T. Severe thrombocytopenia suggesting immunological mechanism in two cases of vivax malaria. Am J Hematol 1997; 56: 183-6.

17. Srichaikul T. Hematologic changes in human malaria. J Med Assoc Thai 1973; 56 (11): 658-63

18. Essien E, Ebhota M. Platelet secretory activities in acute malaria (Plasmodium falciparum) infection. Acta Haematologica 1983; 70: 183-8.

19. Peyron F, Polack B, Lamotte D, Kolodie L, Ambroise-Thomas P. Plasmodium falciparum growth inhibition by human platelets in vitro. Parasitology 1989; 99: 317-22.

20. Polack B, Delolme F, Peyron F. Protective role of platelets in chronic (Balb7C) and acute (CBA/J) Plasmodium berghei murine malaria. Haemostasis 1997; 27: 278-85.

21. Cook BM, Nash GB. Plasmodium falciparum: Characterization of adhesion of flowing parasitized red blood cells to platelets. Exp Parasitology 1995; 80: 116-23.

22. Lou J, Donati YR, Juillard P, Giroud C, Vesin C, Mili N et al. Platelets play an important role in TNF-induced microvascular endothelial cell pathology. Am J Pathol 1997; 151: 1397-405.

23. Dominic K, Adrian V, Idrissa S, Patrick T, John C, Kirk R et al. TNF concentration in fatal cerebral, nono-fatal cerebral and no complicated Plasmodium falciparum malaria. Lancet 1990; 336 (8725): 1201-4.

24. Ockenhouse CF, Magowan C, Chulay JD. Activation of monocytes and platelets by monoclonal antibodies or malaria-infected erythrocytes binding to the CD36 surface receptor in vitro. J Clin Invest 1989; 84: 468-75.

25. Pain A, Ferguson DJ, Kai O, Urban BC, Lowe B, Marsh K et al. Platelet-mediated clumpling of Plasmodium falciparum-infected erythrocytes is a common adhesive phenotype and is associated with severe malaria. Proc Natl Acad Sci USA 2001; 98: 1805-10.

26. Gorski J, Nahorski W, Slonecka A, Birkholz A. Disturbances of hemostasis in malaria. ad Parazytol (resumen) 1993; 39: 339-43.

27. Kakoma I, James MA, Whiteley HE, Montelegre F, Buese M, FafjarWhestone CJ et al. Platelets kinetics and other hematological porfiles in experimental Plasmodium falciparum infections: a comparative study between Saimiri and Aotus monkeys. Kisaengchunghak Chapchi (resumen) 1992; 3: 177-82.

28. Adam C, Geniteau M, Gougerot-Pocidalo M, Verroust P, Lebras J, Gilbert $\mathrm{C}$ et al. Cryoglobulins, circulating immune complexes, and complement activation in cerebral malaria. Infect Immun 1981;31:530-5.

29. World Health Organization WHO. Severe falciparum malaria. Royal Soc Trop Med Hyg 2000;94 Supplement 1.
30. Colombia, Ministerio de Salud. Tratamiento de la malaria. Norma técnica 31/03/2000. Diario oficial (Bogotá) 43.956.

31. López-Antuñano FJ. Diagnóstico microscópico de los parásitos de la malaria en la sangre. En: López-Antuñano FJ, Schmunis G (editores) Diagnóstico de malaria. Washington DC: OPS-OMS; 1988. Publicación Científica 512, p. 39-50.

32. Ault KA, Mitchell F. Analysis of platelets by flow cytometry. En: Darzynkiewic Z, Robinson JP, Harry A. Crissman HA. Methods in Cell Biology: flow cytometry. Vol 42, cap 17. San Diego: Academic Press; 1994 , p. $275-93$.

33. Robinson JP, Darzynkiewicz Z, Dean P, Dressler L, Tanke H, Wheeless L (editores). Handbook of flow cytometry methods. New York: John Wiley \& Sons Inc., 1993.

34. Horstmann RD, Dietrich M, Benzle U, Rarche H. Malaria - induced thrombocytopenia. Blut 1981; 42: 157-64.

35. Horstmann RD, Dietrich M. Haemostatic alterations in malaria correlate to parasitaemia. Blut 1985; 51: 329-35.

36. Baele P, Cormack J, Oldrey T. Thrombocytopenia in malaria with immunoglobulin (IgM) changes. Br Med J 1972; 1: 345-9.

37. 49 Casalino E, Le Bras J, Chaussin F, Fichelle A, Bouvet E. Predictive factors of malaria in travelers to areas where malaria is endemic. Arch Intern Med 2002; 162: 1625-30.

38. 50Wilairatana P, Looareesuwan S. Guideline in management of severe malaria. J Indian Med Assoc 2000; 98: 628-31.

39. Erhart LM, Yingyuen K, Chuanak N, Buathong N, Laoboonchai A, Miller RS et al. Hematologic and clinical indices of malaria in a semiimmune population of western Thailand. Am J Trop Med Hyg 2004; 70: 8-14.

40. Lathia TB, Joshi R. Can hematological parameters discriminate malaria from nonmalarious acute febrile illness in the tropics? Indian J Med Sci 2004; 58: 239-44.

41. Patel U, Gandhi G, Friedman S, Niranjan S. Thrombocytopenia in malaria. J Natl Med Assoc 2004; 96: 1212-4.

42. Scott CS, Van Zyl D, Ho E, Ruivo L, Mendelow B, Coetzer TL. Thrombocytopenia in patients with malaria: automated analysis of optical platelet counts and platelet clumps with the Cell Dyn CD4000 analyser. Clin Lab Haematol 2002; 24: 295-302.

43. Srichaikul T, Pulket C, Sirisatepisan T, Prayoonwiwat W. Platelet dysfunnction in malaria. Southeast Asian J Trop Med Pub Health 1998; 19: 225-33.

44. Mendis KN. Clinical disease and pathogenesis in malaria. Parasitology Today 1995; 11(Pti): 1-PTI16.

45. Chotivanich K, Sritabal J, Udomsangpetch R, Newton P, Stepniewska KA, Ruangveerayuth R et al. Platelet-induced autoagglutination of Plasmodium falciparum-infected red blood cells and disease severity in Thailand. J Infect Dis 2004; 189: 1052-5.

46. Meier S, Krause M, Luthy R, Baumann PC, Markwalder K. Intensive care aspects in severe tropical malaria: clinical aspects, therapy and prognostic factors. Schweiz Med Wochenschr (resumen) 1995; 125: 1033-40.

47. Rogerson SJ, Grau GE, Hunt NH. The microcirculation in severe malaria. Microcirculation 2004; 11: 559-76

48. George JN. Platelets. Lancet 2000; 355 (9214): 1531-39.

49. Bernuau J, Larrey D, Campillo B, Degott C, Verdier F, Rueff B et al. Amodiaquine-induced fulminant hepatitis. J Hepatol 1988; 6: 109 12.

50. Rogier C, Gerardin P, Imbert P. Thrombocytopenia is predictive of lethality in severe childhood falciparum malaria. Arch Dis Child 2004; 89: 795-7.

51. Gerardin P, Rogier C, Gerardin P, Amadous KA, Jouvencel P, Brousse $\mathrm{V}$ et al. Prognostic value of thrombocytopenia in African children with falciparum malaria. Am J Trop Med Hyg 2002; 66: 686-91. 